\title{
CORTO CIRCUITO, AHORA EN LA ERA DIGITAL
}

Isabella Quintero Jaramillo*, Cristian Camilo Yepes López ${ }^{* *}$

\section{Resumen}

La investigación Corto Circuito, ahora en la escena digital, se propuso al percibirse una necesidad en el evento pereirano celebrado mensualmente: Corto Circuito, Escenarios para el Arte de crear piezas promocionales donde se haga una progresión de lo analógico a lo digital y evidenciar su trabajo de manera efectiva. Esto llevado a cabo mediante una investigación-creación cualitativa donde la observación participativa del evento y el registro tanto fotográfico como audiovisual serían los métodos pertinentes de recopilación de datos. Estos permitieron a la investigación crear estrategias de comunicación y emplearlas en contenidos audiovisuales y gráficos funcionales para la promoción y distribución del evento Corto Circuito. Dando en conclusión dos piezas audiovisuales, una campaña gráfica, un sitio web y una cuña radial, todos con un enfoque publicitario y corporativo que expresen la identidad del evento, su audiencia y responder a la necesidad de mantener vivos los espacios socioculturales y artísticos en la ciudad. 


\section{Introducción}

Este proyecto de investigación nace gracias a la celebración de los 15 años del evento cultural Corto Circuito, Escenarios para el Arte, el cual decide involucrar a los estudiantes de Comunicación Audiovisual y Digital de la Fundación Universitaria del Área Andina que participan en el semillero "Memoria y Región", con el objetivo de estudiar, analizar y realizar piezas audiovisuales y gráficas para fomentar la promoción y aumentar la visibilización del evento en los pereiranos, demostrando su impacto en la ciudad.

Mediante un análisis, una conceptualización y una creación de diferentes productos que tengan la finalidad de obtener un posicionamiento y distinción del evento en el sector digital.

Esta investigación pretende entender el comportamiento de la audiencia que ya es asistente como designar cuál es el público objetivo y a cuál evento quiere direccionarse, para así crear canales y mensajes de comunicación efectivos que logren divulgar el evento en la población establecida.

Esta investigación permitirá analizar la condición de un evento de tal talla y a detectar sus debilidades en cuanto a su promoción; la implementación de estrategias creativas efectivas son un camino viable para posicionar cualquier marca, usando narrativas audiovisuales atrayentes, innovadoras y capaces de generar expectativa; analizando cuál es el medio de distribución más funcional y el mensaje más correcto que logre que un espectador se identifique y conecte con la pieza.

\section{Planteamiento del problema}

Durante sus 15 años celebrados en noviembre del 2019, Corto Circuito ha estado vigente y abierto al público, lo que ha formado una audiencia muy comprometida a sus exposiciones y eventos.

En el extenso recorrido del evento ha dejado un legado y un impacto sociocultural en la ciudad de Pereira; demográficamente, el evento fue un emblema para el centro de la ciudad pereirana, gracias a su surgimiento que creó necesidades y preocupaciones en los ciudadanos en aspectos tanto de seguridad para los peatones como de espacio público. Lucía Molina, fundadora del evento y antigua gestora cultural del Colombo Americano, destaca la pertinencia que ha tenido el proyecto desde su comienzo para la sociedad pereirana.

Progresivamente, el evento influenció de manera positiva la perspectiva que se tenía del centro, trajo iluminación y aumentó el flujo peatonal de la zona, promoviendo la cultura y fomentando la seguridad para los ciudadanos. Las exposiciones las realizan de manera activa cinco instituciones y sus gestores culturales: la Alianza Francesa, Colombo Americano, Comfamiliar Risaralda, Fundación Universitaria del Área Andina y la Sala Carlos Drews Castro en el teatro Santiago Londoño. Todas ellas se vinculan formando una ruta o haciendo referencia a su nombre un "circuito", que creaba el recorrido para los asistentes y espectadores para las exposiciones. 
Corto Circuito, Escenarios para el Arte, trae una audiencia muy variada y rica en cuanto a cualidades sociológicas, las cuales no se han estudiado; esto genera que sea complejo formar una comunidad alrededor de Corto Circuito que sobrepase solo a la limitación de asistir a una exposición de arte, sino poder crear un vínculo entre el evento y sus participantes. Debido al tiempo que lleva el evento, ha permanecido así, limitando a los procesos de promoción y expansión del mismo; para ello, es necesario entender su audiencia para la realización de piezas audiovisuales que satisfagan las necesidades de llevar el evento a nuevas poblaciones y se conviertan en asistentes regulares del evento.

\section{Justificación}

Esta investigación nace con el propósito de estudiar, analizar y realizar piezas audiovisuales adecuadas, para dar a conocer la importancia y el impacto que ha generado durante estos 15 años el Corto Circuito, Escenarios para el Arte.

Es necesario promover el evento Corto Circuito, con el fin de visibilizar y mejorar el posicionamiento de esta iniciativa. La investigación propone una serie de piezas audiovisuales y gráficas, las cuales se ubicarán en diferentes plataformas digitales y medios de comunicación, adaptando el evento, cumpliendo con la necesidad que fomenta y predomina en la época actual, de la presencia digital y la inmediatez.

La relevancia de esta investigación consiste en exaltar y promover uno de los eventos culturales más importantes de la ciudad, que hoy forma parte de la agenda cultural de los artistas y de los habitantes de Pereira. Los cuales, durante estos 15 años de trayectoria, han consolidado el impacto de la actividad como una de las más importantes de Pereira y el país; por este motivo, es necesario crear distintas piezas que mantengan visible y fomenten este encuentro mensual.

\section{Objetivo general}

- Producir piezas audiovisuales que promuevan la difusión del evento Corto Circuito, Escenarios para el Arte, el cual es uno de los más importantes en Pereira.

\section{Objetivos específicos}

1. Realizar piezas audiovisuales de carácter ficcional y experimental con finalidad publicitaria.

2. Establecer la imagen del evento en redes sociales.

3. Estudiar el comportamiento y las necesidades de la audiencia de Corto Circuito.

4. Definir los canales apropiados para la distribución de contenidos e información para Corto Circuito.

\section{Metodología}

La metodología empleada para la presente investigación radica en la investigación/creación. Es decir, en el proceso investigativo se incluye no solo el componente teórico, sino también se evalúa y se emplea conocimiento, experiencia, intuición, creatividad e innovación, lo cual genera un producto que no existía y contribuye al crecimiento de la disciplina o caso investigativo; a su vez, 
forma parte fundamental para alcanzar los respectivos objetivos. Esta creación parte de una percepción e interpretación sensible y estética común en las áreas artísticas.

El proyecto de investigación forma parte del grupo DICART, de la Facultad de Diseño, Comunicación y Bellas Artes, del Programa de Comunicación Audiovisual y Digital de la Fundación Universitaria del Área Andina, donde existe el interés de fomentar este método investigativo y, en particular, surge de un interés del colectivo del Corto Circuito, Escenarios para el Arte, para dar a conocer su trabajo ininterrumpido durante 15 años. Los integrantes de las instituciones culturales que constituyen el Corto Circuito, conformaron una alianza con los participantes del Semillero. Este análisis da como resultado la necesidad de plantear una investigación cualitativa, puesto que los fenómenos a analizar presentan variables subjetivas y mutables directamente ligadas a opiniones y percepciones. Para llevar a cabo este tipo de investigación, se implementó diversas técnicas y herramientas previamente seleccionadas para la recolección de datos.

En primer lugar, se conformó el grupo y se asignaron tareas. El cronograma de actividades, en este caso, fue punto de partida para establecer una programación que cubría desde asistencia al evento el segundo viernes de cada mes, búsqueda de archivos, listado de fuentes, cuestionario de preguntas, citas con los gestores y calendario de rodaje. Se dio a la tarea de conocer las dinámicas del Corto Circuito, los promotores, la organización interna, las dificultades y los aciertos del evento, la proyección local y nacional, luego se elaboró un trabajo de campo durante cinco meses, para establecer a través del método de la entrevista cuáles serían las mejores piezas audiovisuales, lo que sirvió para recopilar información ya conocida $y$ previa; a continuación, se implementó la observación participante como técnica principal de recolección de datos.

El trabajo de campo se hizo inicialmente por medio de la asistencia y el registro audiovisual y fotográfico en cada una de las salas que conforman el Corto Circuito ${ }^{*}$, para luego revisar el material y también el insumo visual para la realización de las piezas audiovisuales y gráficas.

Se llevaron a cabo entrevistas tanto formales como casuales a diversas fuentes que presentan un apoyo informativo para la conceptualización de las piezas y el desarrollo de investigación. Se hicieron un total de quince entrevistas a los seis gestores de cada sala de manera individual, cuatro artistas en diferentes disciplinas y cinco asistentes a los eventos de diferentes características poblacionales. El proceso de creación de los productos resultados del proyecto de investigación, se efectuó en paralelo con el trabajo de campo, obteniendo la propuesta de una campaña gráfica publicitaria con diversas piezas, un video corporativo y la página web ${ }^{\star *}$ del evento Corto Circuito, lo que permitió la visibilización de los procesos artísticos que en él se reflejan.

* Este se realizaba el segundo viernes de cada mes.

* https://cortocircuitoperei.wixsite.com/ cortocircuito/ 


\section{Diseño del estudio}

Para el diseño del actual proyecto, el equipo de trabajo propuso una revisión consignada en los periódicos de la ciudad de Pereira (La Tarde y El Diario del Otún), desde los inicios del Corto Circuito, en el 2005 hasta el 2020. Esa revisión consistió en comentarios, reseñas y artículos que se refirieran al evento cultural.

El otro frente de trabajo fue acercarse, hablar y entrevistar a los gestores del Corto Circuito, los cuales ofrecieron su testimonio y su mirada histórica a cerca de la importancia y el impacto del evento para las distintas manifestaciones artísticas de la ciudad de Pereira y del país.

\section{Discusión}

Se encontró un evento con mayor acogida de la esperada, con una trayectoria que genera impacto y un sin fin de oportunidades para los espacios culturales y sociales en la ciudad de Pereira, gracias a sus diferentes salas de exposición y la divulgación de diversos tipos de expresiones artísticas.

La audiencia, es un factor primordial para mantener vivo el evento, donde no solo se necesita entenderla, sino también hacer caso a sus necesidades para ofrecerle una experiencia completa.

Teniendo en cuenta la poca visibilización y analizando esta desventaja, se encuentra la falta de presencia en lo digital, dejando por fuera un gran sector de posible público, perdiendo la oportunidad de fidelizar a la audiencia y encontrar nuevos sectores para explorar y ayudar a engrandecer este evento.
También, el reconocimiento de grandes ventajas como la cantidad de artistas involucrados y el profesionalismo de sus gestores, ha generado una imagen favorable en el medio.

Después de un primer acercamiento a las redes sociales, se determinó la gran acogida que tuvo para el público y la importancia de seguir migrando y cultivando este medio.

Difundir de manera correcta el valor del evento Corto Circuito, Escenarios para el Arte, teniendo en cuenta que los canales de distribución son los más apropiados para llegar a su audiencia y qué información es pertinente para afianzar tanto a la audiencia regular como a una nueva audiencia.

Hasta el momento de la investigación, que comenzó en agosto del 2019, se ha logrado entablar conversación mediante entrevistas formales con los cinco actuales gestores de cada sala para conocer el panorama actual del evento. Se asiste mensualmente a las exposiciones para observar de primera mano la población actual del evento, reconocer y entender sus cualidades, necesidades y fortalezas.

Se ha explorado el recorrido del evento históricamente para entender mejor su trayectoria, escuchando testimonios, entrevistando y evidenciando la interacción social que genera entre artistas y público. Una de las estrategias más funcionales es el análisis del material de archivo que incluye evidencia de la constancia del evento.

Después de un primer acercamiento a las redes sociales, se determinó la gran 
acogida que tuvo para el público y la importancia de seguir migrando y cultivando este medio.

\section{Sugerencias}

Corto Circuito, Escenarios para el Arte, es un evento muy estable en la ciudad de Pereira que, a pesar de su extenso recorrido, ha tenido dificultades y deficiencias a la hora de promocionarse y usar diferentes medios para ello, lo que ha sido la razón de la poca visibilización.

El evento cultural, Corto Circuito, Escenarios para el Arte, se limita a los medios de difusión análogos, como su tarjeta de presentación mensual obsequiada en el evento, abarcando la mayoría de veces el público ya afianzado y una población más empírica al momento de su publicidad; por tanto, no se abordan nuevas audiencias externas, las cuales podrían ser nuevos asistentes.

Como parte de la investigación, nació la necesidad de entender los diferentes factores de la audiencia ya obtenida para replicar estas mismas cualidades en la población, a la que se le quería direccionar la promoción.

Gracias a la recolección de información obtenida mediante la observación, se logra comenzar a diseñar un plan de medios y de estrategias comunicativas para solucionar el problema planteado.

La observación hecha al evento, su gestión, sus exposiciones y sus asistentes, se concluye que usar canales tanto aná$44 \operatorname{logos}$ en las estrategias de distribución forma parte fundamental del proceso de promoción, como la radio, haciendo uso de emisoras de la ciudad de Pereira y del Eje Cafetero, ya que permite la fidelización de la audiencia más adulta como el uso de canales digitales.

La implementación de redes sociales como canales oficiales del evento, también es fundamental para la distribución de contenidos tanto audiovisuales como spots publicitarios y piezas gráficas que permitan interacción, divulgación y tráfico en una audiencia más allegada a la virtualidad.

\section{Impacto}

Esta investigación tiene un impacto positivo en factores tanto culturales como sociales en la ciudad de Pereira, ya que se denotará la importancia y el valor de la propuesta que tiene el evento Corto Circuito, la cual es mostrar el desempeño artístico y la capacidad creativa no solo en la ciudad, sino también en el país.

Mediante las piezas audiovisuales se reconocerá cómo este evento ha sido un distintivo en cuanto a las actividades para la ciudad de Pereira en el contexto artístico, puesto que su extenso tiempo vigente $\mathrm{y}$ al impacto social genera traer artistas de toda índole, reunir sedes educativas y abrir las puertas de un espacio sano a todos sus asistentes.

El impacto social radica en que se difundirá un espacio cultural importante para la ciudad, y personas de todo tipo podrán asistir a él de manera gratuita.

El impacto que generará también se refleja en las personas con vínculos con el evento, 
ya que la difusión de su proyecto se hará más evidente y de manera más eficaz. A su vez, el público fidelizado al evento encontrará un espacio oficial de información.

Se reforzarán los ideales de Corto Circuito de atraer nuevas personas y que más personas conozcan la importancia del evento y el ambiente cultural, que se mueve alrededor de esto para una extensa cantidad de personas.

También se logrará una fidelización de la audiencia de Corto Circuito. Ellos tendrán espacios oficiales donde puedan interactuar e informarse de manera verídica.

\section{Agradecimientos}

En primer lugar, expresamos nuestro agradecimiento al asesor de este semillero, docente, investigador y periodista Franklyn Molano Gaona, por su dedicación y liderazgo dedicado a este proyecto, por siempre permitirnos explorar como comunicadores y siempre guiarnos de la mejor manera. Gracias por haber puesto ese gran voto de confianza en nosotros y cultivar nuestro proceso formativo en todo nuestro proceso estudiantil.

Le agradecemos a nuestra institución educativa, Fundación Universitaria del Área Andina, seccional Pereira, por formarnos de manera constante e inagotable, por poner todos sus recursos tanto humanos como su equipo docente y recursos tecnológicos para formarnos cada día como profesionales integrales, capaces de asumir y superar riesgos.

Por su disposición y entrega agradecemos a Jorge Mario Quintero, coordi- nador cultural de la Alianza Francesa de Pereira; Sandra Milena Martínez, directora cultural del Centro Colombo Americano; Gustavo Colorado, asesor cultural de Comfamiliar; James Llanos, curador de la Sala Carlos Drews Castro; Nelly Herrera Vargas, coordinadora de la Biblioteca Otto Morales Benítez, y Lucía Molano, fundadora del evento. Por habernos abierto las puertas de sus valiosas instituciones, permitiendo que el proyecto fluyera de la mejor manera, por habernos compartido tanto conocimiento y envolvernos en el mundo artístico y cultural. A los artistas que mes a mes comparten su talento y a los visitantes de las salas que mantienen vivo el evento y resaltan la importancia de los espacios culturales en la ciudad.

Agradecemos de manera individual a nuestras familias que fueron testigos de la entrega y dedicación puesta para la investigación y nunca dejarnos rendir, especialmente a nuestras madres que son un motor para cada paso que damos.

Pero, sobre todo, nos agradecemos el uno al otro, por ser un pilar fundamental para la realización de este proyecto y siempre prestarnos un gran apoyo moral y humano, necesarios en los momentos difíciles de este trabajo.

\section{Referencias}

1. La tesis de grado Los museos y el arte de la seducción, un recorrido para enamorarse de la cultura de Ana María Rocha Pardo, Natalia Roldán Rueda (2008).

Analiza mediante diversos acontecimientos artísticos las causas generales del interés en el arte en los individuos, a tal punto de crear un movimiento, y asimismo una comunidad a partir de ellos. Para en- 
tender dichas manifestaciones artísticas y del mismo modo fomentarlas, se demuestra en el estudio las debilidades y fortalezas del público y sus expositores. Como objeto de estudio en este caso, los museos pasan a ser parte fundamental para la comprensión de dichas interacciones.

2. Artículo Vea lo mejor del arte universitario en el Museo de Arte Contemporáneo, Revista El Tiempo (2018).

El Museo de Arte Contemporáneo de Bogotá presenta los trabajos de grado ligados a las artes plásticas y visuales de todo el país, plasmando la pertinencia de espacios artísticos y como llegan a ser oportunidades académicas y educativas para los públicos. Dando visibilidad e importancia a las manifestaciones que involucren artes plásticas y visuales. Además, resalta la importancia del vínculo que se crea con los escenarios que se disponen para el arte, promoviendo nacionalmente este movimiento.

3. La tesis de grado Museo de las Artes Locales de Sara Elizabeth Tinoco Vera (2013). Resalta la esquematización generada en las ciudades, dando prioridad a los marcos administrativos, culturales e institucionales. Plasma a Bogotá, en este caso, como una ciudad versátil que no se ha dejado vencer por esos esquemas y ha logrado interesar al público nacional e internacional con espacios innovadores y transformables. En concreto, el proyecto INNOBO, que consta de ferias, eventos y convenciones para incentivar estos movimientos y demostrar la importancia de los mismos.
4. La tesis de grado Influencia de las estrategias publicitarias utilizadas en el evento artístico Música en La Perla como aporte al desarrollo cultural de la ciudad de Guayaquil en el año 2017 de Óscar Alfredo Gurumendi León (2017). Un proyecto que pretende visibilizar las expresiones artísticas y reforzar su imagen mediante estrategias publicitarias, donde se analizan y entiende la necesidad de promover y utilizar herramientas que resaltan la importancia de estos espacios, con varios objetivos como aumentar la interacción.

5. La tesis de grado Estrategia de comunicación aplicada a empresas organizadoras de eventos de Fadya Halyme Mahamud Acevedo (2016). Este trabajo de grado expone estrategias de comunicación digital enfocado en eventos y de igual manera expone la importancia que tienen las nuevas tecnologías para la promoción y fidelización de la respectiva audiencia del evento. 